\title{
Image Localization Using Google Maps
}

\author{
Sadashiv D. Lavange ${ }^{1}$, A. D. Vishwakarma ${ }^{2}$, H. T. Ingale ${ }^{3}$, V. D. Chaudhari ${ }^{4}$ \\ ${ }^{1}$ GF's Godavari College of Engineering, NM University Jalgaon, India \\ ${ }^{2,3,4}$ Professor, GF's Godavari College of Engineering, NM University Jalgaon, India
}

\begin{abstract}
Image localization and image registration techniques are the mostly used by the researchers for searching image in specified place. Google earth image searching is required for localization and analysis of specific area over the areal image. This type of searching is useful for calculating the area of solar rooftop in any specific location like Jalgaon city. Within the range of city, it is required to get snap shots of areal images within which rooftop is required to search. This project is proposed for the search of template area over the areal map area. We have designed the algorithm, in which input is Latitude and Longitude of the specific location and the template of image say (roof top of a building) to be searched at that specific location and the output of the algorithm is the localization of the template area over big areal image. To identify the Latitude and Longitude of the specific area, various apps are available which provides the information about Latitude and Longitude by providing the area name. This information is useful to this algorithm for localization of the specific area. The challenges are 1) to get the Google earth image based on the Latitude and Longitude 2) and template registration on the Google map image. We used MATLAB R2009a for getting Google earth image specific to the given Latitude and Longitude. And 2-D cross-correlation is proposed for registration of template on the Google map image.
\end{abstract}

Keywords: Image localization, Latitude and Longitude, Template Image, Areal Image

\section{Introduction}

Many researchers have used image localization and image registration techniques for identifying the image in specified place. Image localization provides the location of places over the specific image and image registration means the localization of template image over large image through localization, image warping of template image for exact matching like the identified image over the large image. This application requires the localization of template over the large image called areal image. For localization and analysis of specific area over the areal image, Google earth image searching is proposed which may be useful for automatic calculation of area over the specific location on the Google earth.

Now days, government of India is making awareness about green energy. So, various analyses have to make for promotion of green energy like solar energy over the country. It is required to know about the rooftop area useful for holding solar panels. Proposed algorithm can be used for identifying this type of area through satellite images with the use of Google earth services. With the use of Google earth services, we can get various snap shots of areal images with in the large range of area like a city of Jalgaon. Once we get the areal images the task remaining is to identify the target area called template over the large image. Localization of template over the large area is proposed in this project.

Using Google earth services, it is possible to enlist the Latitude and Longitude of the specific location very easily. Various apps are available for getting Latitude and Longitude of the specific locations. Various Google web services are also available for this purpose. Algorithm is proposed in which the area to be localized is mapped over the given large areal image by providing Latitude and Longitude of the specific location and the template of image say (roof top of a building) and the template of image say (roof top of a building) to be searched. Template may the small image showing the roof top of the specific building. And areal image must be larger image in which the template view is available at anywhere on the areal image.

Google earth services are available to get Google earth image but we designed the MATLAB code for using this service in which it is required to get Google service API KEY for the access that service using MATLAB. By using API KEY in MATLAB code, it is possible to read/save the images from Google web services using web read (url) command (MATLAB R2009a). 2-D cross-correlation is proposed for localization of template over the Google map image. Images require to be used for 2-D cross-correlation function are gray scale images as the processing is only possible on 2-D data, so colour to gray scale image conversion is used.

\section{Literature Survey}

Yang Long, Yiping Gong, Zhifeng Xiao, and Qing Liu et.al [1], introduced the tackling the problem of automatic accurate localization of detected objects in high resolution remote sensing images. The difference between object detection and object localization is subtle. Object detection focuses on detecting the presence of entire objects. But object localization has higher requirements than object detection does. Object localization requires that objects be located accurately. They propose an accurate object localization framework for remote sensing images.

Amir Roshan Zamir and Mubarak Shah, et.al [2], Finding an image's exact GPS location is a challenging computer vision problem that has many real-world applications. In this paper, address the problem of ending the GPS location of images with an accuracy which is comparable to hand-held GPS devices. First, each image is localized individually; then, the rest of the images in the group are matched against images in the neighboring area of the found match. The location is determined based on the Localization parameter. The proposed image group localization method can deal with very

\section{Volume 6 Issue 7, July 2017}




\section{International Journal of Science and Research (IJSR) \\ ISSN (Online): 2319-7064}

Index Copernicus Value (2015): 78.96 | Impact Factor (2015): 6.391

unclear queries which are not capable of being geo located individually. In this paper we addressed the problem of ending the exact GPS location of images.

Zheng Wang \& Faisal Z. Qureshi et.al [3],developed it is possible to geo-localization images using existing geo-tagged images by exploiting visual similarities between the query images and one or more geo-tagged images. Zamir and Shah presented a system capable of using low-level image features to geo-localize an image using Google Maps Street View data. Our method learns a topic model over the reference database, which in turn is used to divide the reference database into scene groups. Each scene group consists of "visually similar" images as determined by the topic model. We present a method for partitioning a geo-tagged reference image database into scene groups.

Changchang Wu, Friedrich Fraundorfer, Jan-Michael Frahm, Jack Snoeyink and Marc Pollefeys et.al [4], designed method to index ortho-map databases with image-based features and search a map database for regions that match query images of unknown scales and rotations. The proposed method uses image-based features to index the 2D map locations Image. In this paper, we are interested in the problem of matching aerial images (with unknown scales and rotations) to a map database. Given a particular aerial image, we propose a method to find the locations of similar map data along with the relative scales and rotations, and provide a confidence measurement for the similarity. The paper proposed a new visual word for indexing orthogonal satellite imagery and the associated method for image-based localization

Pratik Agarwal Wolfram Burgard Luciano Spinello et. al [5], present a novel approach that instead uses geotagged panoramas from the Google Street View as a source of global positioning In this paper, we present a novel approach to metric localization by matching Google's Street View imagery to a moving monocular camera. Our method is able to metrically localize without requiring a robot to pre-visit locations to build a map where Street View exists. The model the problem of localizing a robot with Street View imagery as a non-linear least squares estimation in two phases. The first estimates the 3D position of tracked feature points from short monocular camera streams, while the second computes the rigid body transformation between the points and the panoramic image.

Hang Chu Hongyuan, Mei Mohit Bansal, Matthew R. Walter et.al [6], propose a method for accurately localizing ground vehicles with the aid of satellite imagery. Our approach takes a ground image as input, and outputs the location from which it was taken on a georeferenced satellite image. They propose a strategy for localizing a camera based upon an estimate of the ground image-satellite image co-occurrence, which improves localization accuracy without ground image database expansion. We describe a ranking-loss method that learns general feature projections to effectively capture the relationship between ground and satellite imagery. Our approach first constructs a ground-satellite feature views using an existing database from the area. Second, we learn projection matrices for the each of the two views so as to arrive at a feature embedding that is more location discriminative.

Georgios Floros, Benito van der Zander, and Bastian Leibe et..al [7], describe Localization is an important component regarding the stability of such systems and thus its precision and robustness is of high importance. Current localization methods rely mostly on GPS information. However, GPS signals are not always available (e.g., inside narrow streets) and are usually only accurate up to a range of several meters. To overcome this problem, several authors have proposed to use image information from camera systems mounted on top of the vehicles. They have presented a novel approach for globally localizing a vehicle's position. Our approach combines a classical visual odometry pipeline with map data from Open Street Maps in a unified framework, achieving improved performance on a set of challenging sequences. We have described the algorithmic and implementation details of our method and they have thoroughly evaluated it using data from a demanding benchmark. Our results show that the proposed system is able to provide fully automatic global localization at a low infrastructural cost (only street graphs are necessary) and that it is able to stabilize odometry against drift over long travel distances. In the future we plan to further investigate the use of maps to facilitate several other tasks, such as understanding of the environment and benefiting from the large amount of additional information that is stored in such maps.

\section{Methodology}

\subsection{System Operation}

This Google earth image searching is required for localization and analysis of specific area over the areal image. This type of searching is useful for calculating the area of solar rooftop in any specific location. Within the specific range, it is required to get snap shots of areal images within which rooftop is required to search. This project is proposed for the search of template area over the areal map area. Input is Latitude and Longitude of the specific location and the template of image to be searched at that specific location. To identify the Latitude and Longitude of the specific area, various apps are available which provides the information about Latitude and Longitude by providing the area name. This information is useful to this algorithm for localization of the specific area.

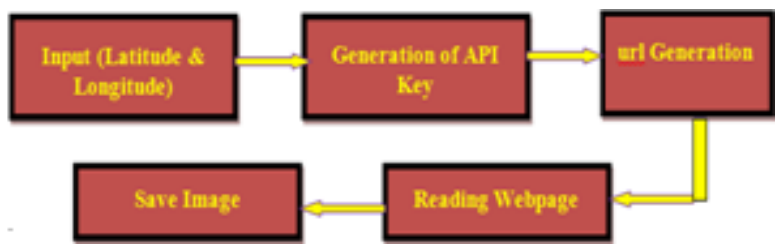

Figure 1: Block Diagram to get Image from Google earth

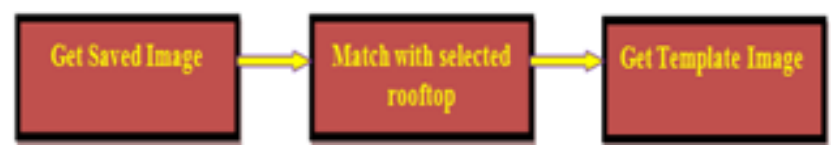

Figure 2: Block Diagram to get template image 


\section{International Journal of Science and Research (IJSR) \\ ISSN (Online): 2319-7064}

Index Copernicus Value (2015): 78.96 Impact Factor (2015): 6.391

The above figure 1 develops this system for getting the required image from the Google earth. As we mention the latitude \& longitude from the web and enter in the system then it generate the required image. And from this specified image, if we highlight some portion of it, then we also locate this highlight portion in the given image say as template image as indicating the above figure 2 .

\subsection{System Flow Chart}

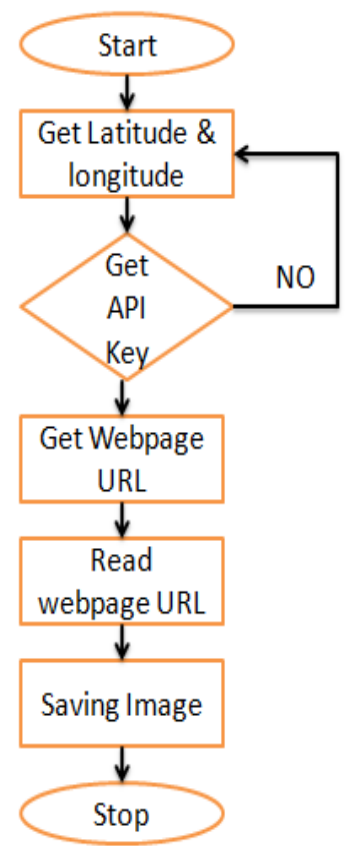

Figure 3: Flow Chart for getting image

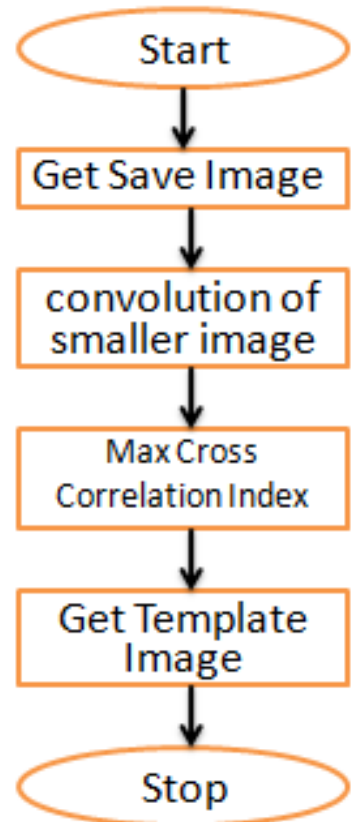

Figure 4: Flow Chart for template image

\subsection{Algorithm Steps}

I) Steps required for getting the Google earth image based on the Latitude and Longitude is,

1) Get Latitude and Longitude of the given location say Godavari College of Engineering Jalgaon.
2) Get the template image as a top view of areal image manually taken from map service of Google.

3) Get API KEY for Google earth service.

4) Get web page url for Google earth service so that web page is showing location of given Latitude and Longitude. For this define the web url using MATLAB specifying the different parameters like Latitude, Longitude, zoom index etc.

5) Read the webpage using webread(url) command to display the web page in default browser.

6) Save the image (.png format) in webpage as areal image corresponding to the specified Latitude and Longitude.

II) Steps required for 2-D cross-correlation function are,

1) Get template image and areal map image.

2) Convert both the images in gray scale i.e. into 2-D data.

3) Perform convolution of the smaller image i.e. template image over the large image i.e. map image. Convolution function includes 2-D cross-correlation function so that if information along the convolution area is matched, it provides higher value as a cross-correlation index.

4) Find the maximum cross-correlation index and its $x$ and $y$ coordinates indicates the location of matted portion.

\section{Results}

\subsection{Final output}

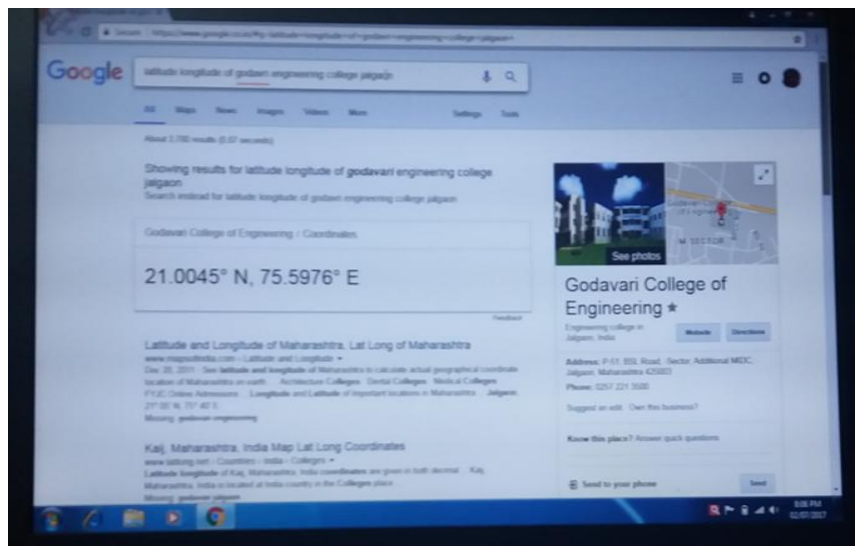

Figure 5: Latitude \& Longitude of Godavari College from Web

Figure 5 shows the Latitude \& Longitude of Godavari Engineering College From Web for specific image searching. With the help of Matlab software, we located the image by taking latitude \& longitude of specific location from the Google. After locating the image we highlighted the portion i.e. template image which is required. Following are the different result for various location of the image with the help of their latitude \& longitude. This latitude \& longitude are provided by various app or web. After searching of location of image, we highlight the required part of image say as template image.

1) Top view of Godavari College of Engineering is taken manually from Google earth. And from location using Latitude and Longitude is identified with the use of MATLAB code. The areal image is saved and used for 2 


\section{International Journal of Science and Research (IJSR) \\ ISSN (Online): 2319-7064}

Index Copernicus Value (2015): 78.96 | Impact Factor (2015): 6.391

D cross-correlation function to map template on areal image as shown in following images.

Latitude $=21.00450 \mathrm{~N}$

Longitude $=75.59760 \mathrm{E}$

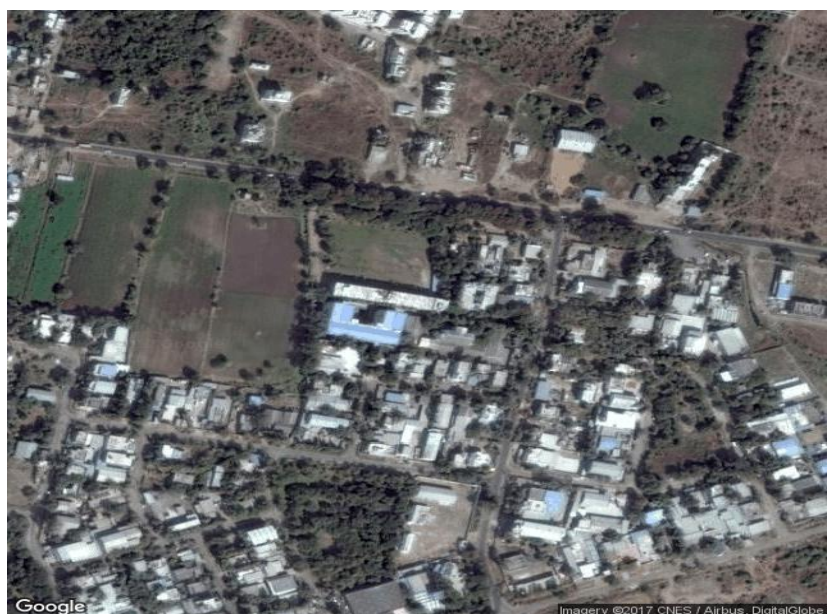

Figure 6: Areal map Image of Godavari Engg College

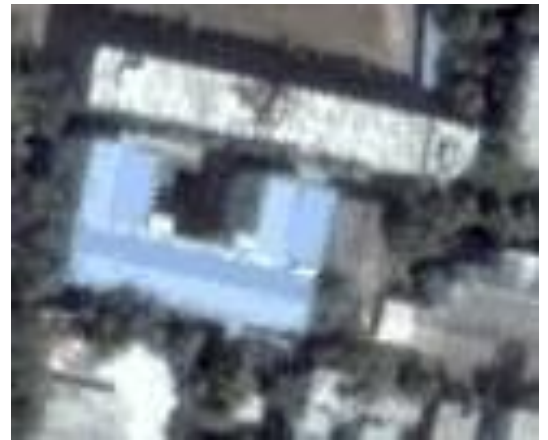

Figure 7: Template Image of Godavari Engg College

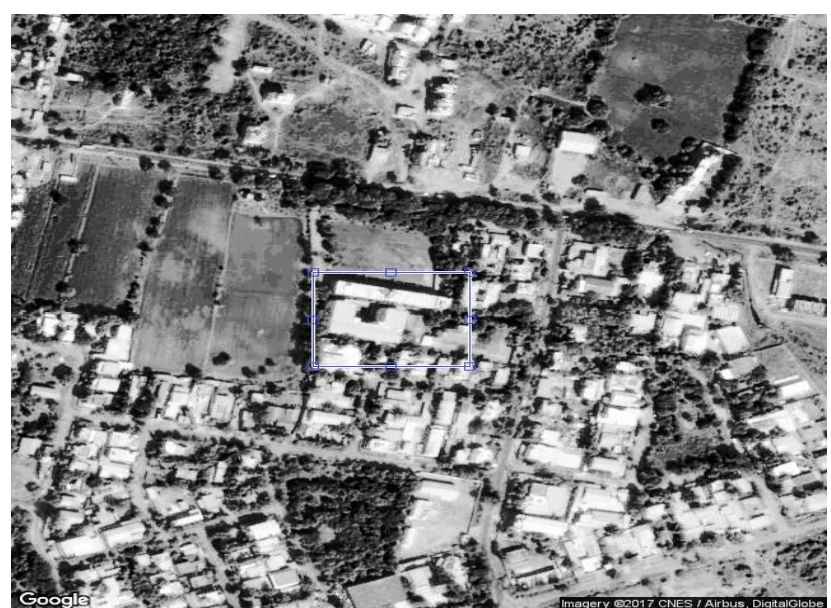

Figure 8: Located Image of Godavari Engg College

2) Top view of North Maharashtra University is taken manually from Google earth. And from location using Latitude and Longitude is identified with the use of MATLAB code. The areal image is saved and used for 2$\mathrm{D}$ cross-correlation function to map template on areal image as shown in following images.

Latitude $=21.00330 \mathrm{~N}$

Longitude $=75.49390 \mathrm{E}$

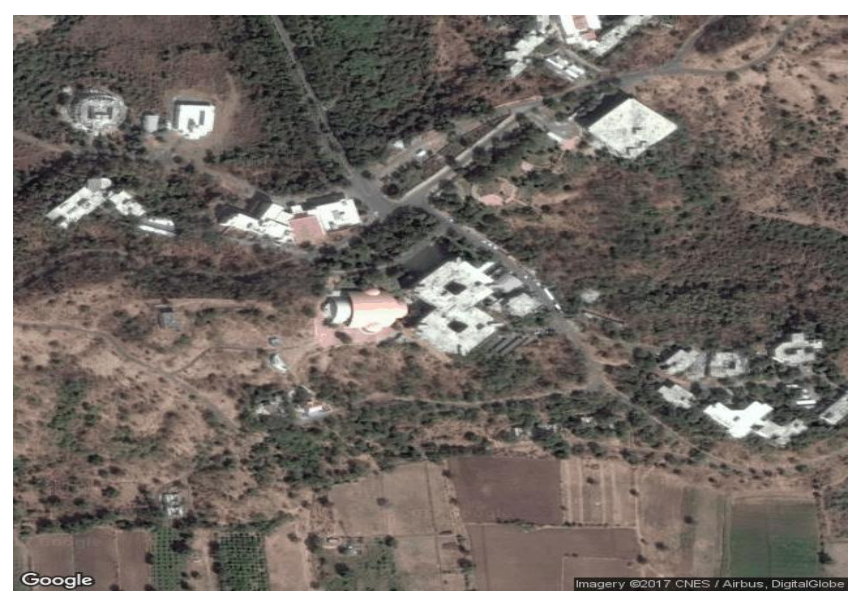

Figure 9: Areal Map Image of North Maharashtra University

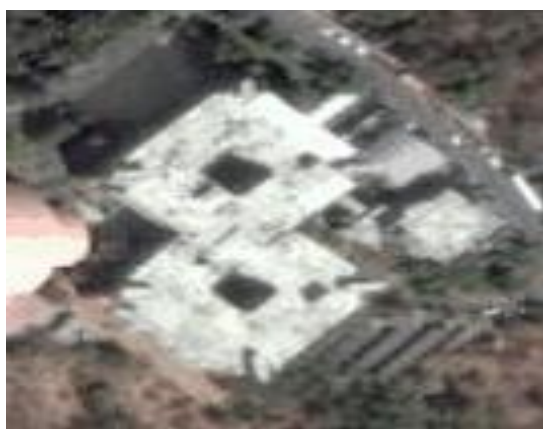

Figure 10: Template Image of North Maharashtra University

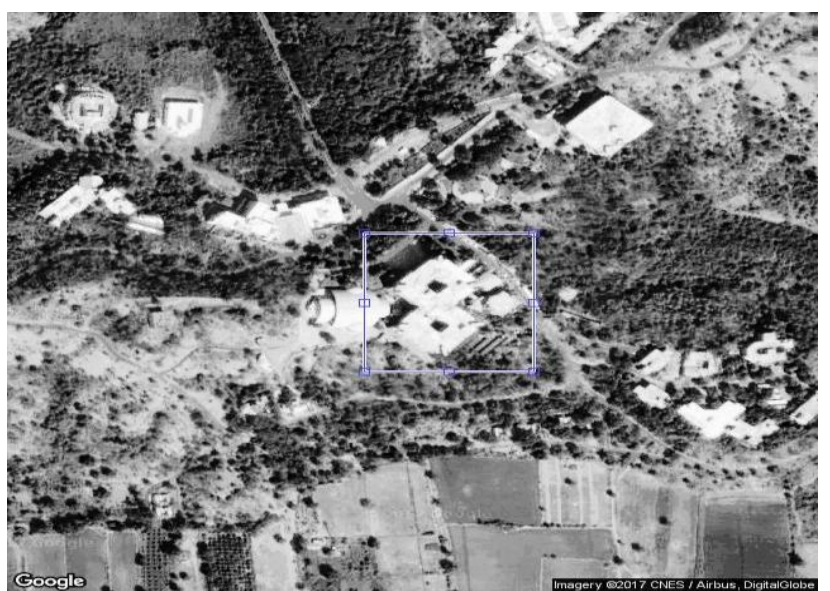

Figure 11: Located Image of North Maharashtra University

\subsection{Result Analysis}

The analysis of above result is given in below table.

Table 1: Result analysis of different location

\begin{tabular}{|c|c|c|c|c|}
\hline S. $N$. & Location & Latitude & Longitude & $\begin{array}{c}\text { Result } \\
\text { Image }\end{array}$ \\
\hline 1 & $\begin{array}{c}\text { Godavari Engineering } \\
\text { College }\end{array}$ & $21.0045^{0} \mathrm{~N}$ & $75.5976^{0} \mathrm{E}$ & $\sqrt{ }$ \\
\hline 2 & $\begin{array}{c}\text { North Maharashtra } \\
\text { University }\end{array}$ & $21.0033^{0} \mathrm{~N}$ & $75.4939^{0} \mathrm{E}$ & $\sqrt{ }$ \\
\hline 3 & Jalgaon Railway Station & $21.0182^{0} \mathrm{~N}$ & $75.5630^{0} \mathrm{E}$ & $\sqrt{ }$ \\
\hline 4 & $\begin{array}{c}\text { Bhusawal Railway } \\
\text { Station }\end{array}$ & $21.0470^{0} \mathrm{~N}$ & $75.7884^{0} \mathrm{E}$ & $\sqrt{ }$ \\
\hline 5 & Akola Bus Stand & $20.7077^{0} \mathrm{~N}$ & $77.0044^{0} \mathrm{E}$ & $\sqrt{ }$ \\
\hline
\end{tabular}




\section{International Journal of Science and Research (IJSR) \\ ISSN (Online): 2319-7064}

Index Copernicus Value (2015): 78.96 Impact Factor (2015): 6.391

\section{Conclusion}

Localization of Google earth image is proposed for template matching. The results show the validation of two algorithms proposed in this work. Getting Google earth image corresponding to given Latitude and Longitude is a specific work performed in this work. Template matching is also the main task performed to identify the exact location and results we got are as per the requirement.

We have designed a system for searching the images from the Google earth \& get the exact position of any location present in the searching image. We also consider the some of the future scope of this system as the Google earth images are clear in pixel identification; we got the clear image of specific location. Also in case we will find out in assigned location the object which will be repeated in this region and how many time it is repeated in given location. Therefore by considering the future scope, this system will be used in military application as well industry base application.

\section{References}

[1] Yang Long, Yiping Gong, Zhifeng Xiao, and Qing Liu, "Accurate Object Localization in Remote Sensing Images Based on Convolutional Neural Networks", IEEE Transactions On Geoscience And Remote Sensing, Vol. 55, No. 5, May 2017.

[2] Amir Roshan Zamir and Mubarak Shah, "Accurate Image Localization Based on Google Maps Street View", University of Central Florida, Orlando FL 32816, USA. June 2014.

[3] Hang Chu Hongyuan, Mei Mohit Bansal, Matthew R. Walter, "Accurate Vision-based Vehicle Localization using Satellite Imagery “.May 2013.

[4] R. Rajbanshi, Q. Chen, A.Wyglinski, G. Minden, and J. Evans, "Quantitative comparison of agile modulation technique for cognitive radio tranceivers," in Proc. IEEE CCNC, Jan. 2007, pp. 1144-1148.

[5] Georgios Floros, Benito van der Zander, and Bastian Leibe, "OpenStreetSLAM: Global Vehicle Localization Using Open Street Maps”. April 2016.

[6] Tsung- Yi Lin and Serge Belongie \& James Hays, "Cross-View Image Geolocalization". July 2012.

[7] Jesse Levinson, Michael Montemerlo, Sebastian Thrun, "Map-Based Precision Vehicle Localization in Urban Environments".,May 2015.

[8] Yannis Kalantidis ,Giorgos Tolias, Yannis Avrithis, Marios Phinikettos, Evaggelos Spyrou , Phivos Mylonas, Stefanos Kollias, “ VIRaL: Visual Image Retrieval and Localization”, October 22, 2010.

[9] Yannis Kalantidis, Giorgos Tolias, Evaggelos Spyrou, Phivos Mylonas, Yannis Avrithis, "Visual Image Retrieval and Localization",Feb 2016.

[10] Florin-Andrei Georgescu, , Dan R aducanu, and Mihai Datcu, Fellow, "New MPEG 7 Scalable Color Descriptor Based on Polar Coordinates for Multispectral Earth Observation Image Analysis", ieee geoscience and remote sensing letters, vol. 14, no. 7, july 2017.
[11] Torsten Sattler, Bastian Leibe, Leif Kobbelt, “ Fast Image-Based Localization using Direct 2D-to-3D Matching", publication in 2011 IEEE.

[12] S.N.A. Vlaszaty \& T.R.L. Timmermans, " Image-based localization of pictures using Google Street view images", February 13, 2013. 